\title{
The role of Cryptococcus neoformans Cys-3 transcription factor in sulfur uptake
}

Amanda Teixeira de Melo; Kevin Felipe Martho ${ }^{1}$; Alexandre K. Tashima ${ }^{2}$; Marcelo A. Vallim ${ }^{1}$; Renata C. Pascon ${ }^{1}$ 1 Universidade Federal de São Paulo, Campus Diadema, Laboratório de Interações Microbianas, Diadema, São Paulo, Brazil. 2Universidade Federal de São Paulo, Campus São Paulo, Brazil.

In order to adapt to diverse ecological niches, such as the animal host and environment, $C$. neoformans, an opportunistic pathogen, relies on its ability to uptake nutrients, such as nitrogen, sulfur and amino acids. We studied the sulfur assimilation pathway and its implications on $C$. neoformans biology. The gene CNAG_04798 annotated in the genome as CYS3, encodes a BZip transcriptional factor, which has been considered an essential gene. In this work we showed that Cys-3 is only essential in rich medium; its deletion led to sulfur amino acids auxotroph, which can be satisfied only by supplementation with cysteine (Fig. 1A). Furthermore, the deletion affected the growth of the mutant under alkaline and cell wall stress (Fig. 1B). Fluorescent microscopy showed that a GFP-Cys-3 (CNU080) co-localizes with DAPI staining in the nucleus during growth in YEPD (80 $\pm 3 \%)$, irrespectively of the temperature; co-localization is reduced in nitrogen deprived medium $(10 \pm 4 \%)$ at $30^{\circ} \mathrm{C}$ and at higher temperature growth reaches $(1 \pm 0.5 \%)$, however this difference is not statistically significant (Fig. 2A and B). Western blot analysis showed that Cys-3 has phosphorylated and dephosphorylated patterns (Fig. 2C), which varies according to nutritional condition (Fig. 2D). GFP-Cys-3 was used in immunoprecipitation assays and a protein complex was found by MALDI-TOF and LC/MS. Three interacting phosphatases were identified: CNAG_01744 (GPP2), CNAG_04796 (calcineurin-CNA1) and CNAG_03370 (CNB1). Two-hybrid assay confirmed the interactions found by proteomics (Fig. 3) and gene deletion experiments in the GFP-Cys-3 background (CNU080) revealed that deletion of calcineurin leads to Cys-3 abnormal dephosphorylation pattern and miss localization of the protein to the cytoplasm in YEPD (Fig. 4). Deletion of Gpp2 promoted hyperphosphorylation of Cys-3 in nitrogen deprived medium and persistent co-localization with DAPI (Fig. 5), suggesting that Cys-3 is the target of Gpp2, which in turn is the target of calcineurin complex (Fig. 7). Moreover, qPCR showed that sulfate transporter Sul1 is induced in YEPD and repressed in SD (Fig. 6A). On the other hand, sulfur amino acid permease Mup1 is induced in SD $-\mathrm{N}$ and repressed in YEPD (Fig. 6B). The knowledge of this process and its impact on virulence can bring significant understanding on the mechanisms of invasive fungal infections.

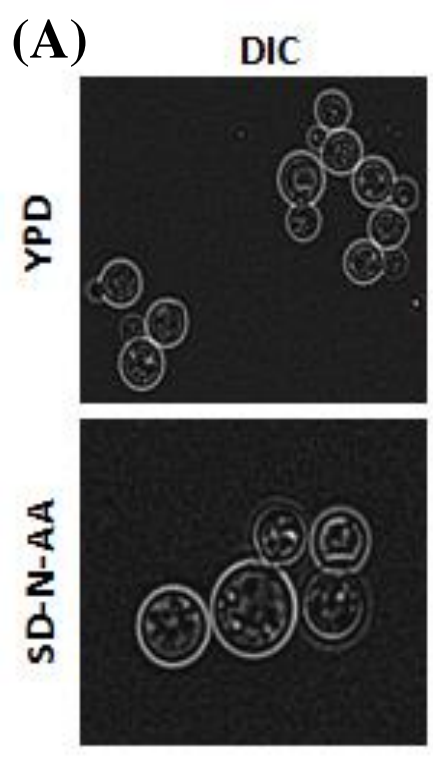

(C)
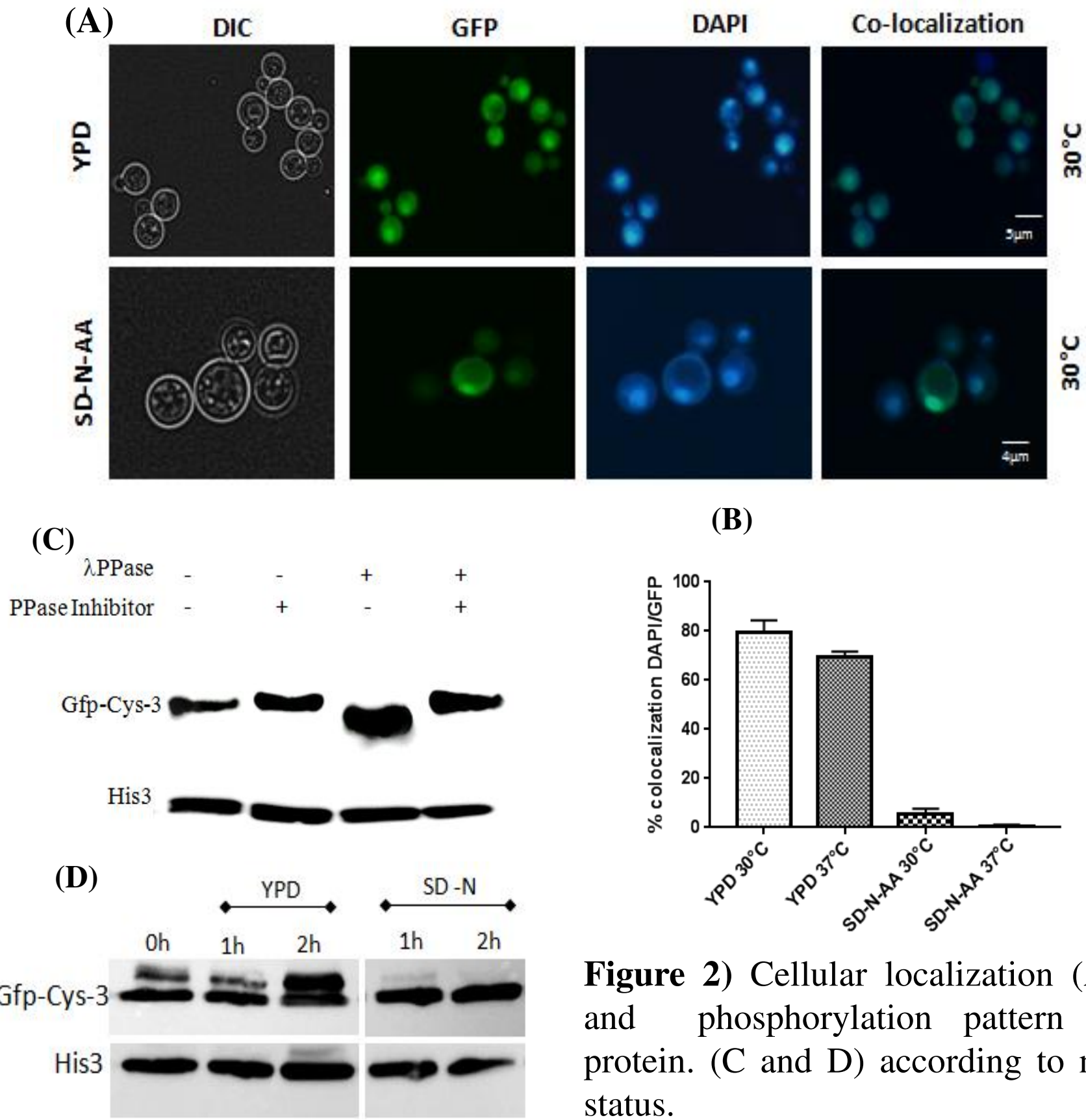

(B)

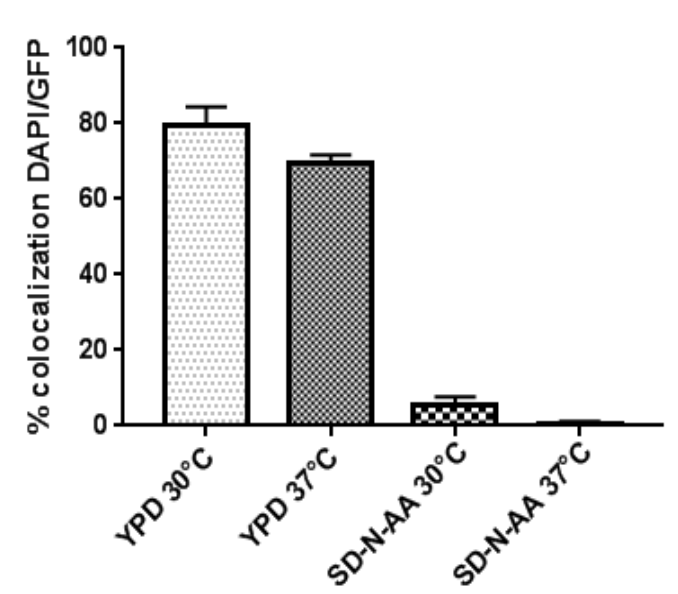

Figure 2) Cellular localization (A and $\mathrm{B}$ ) and phosphorylation pattern of Cys3 protein. (C and D) according to nutritional status.

(A)

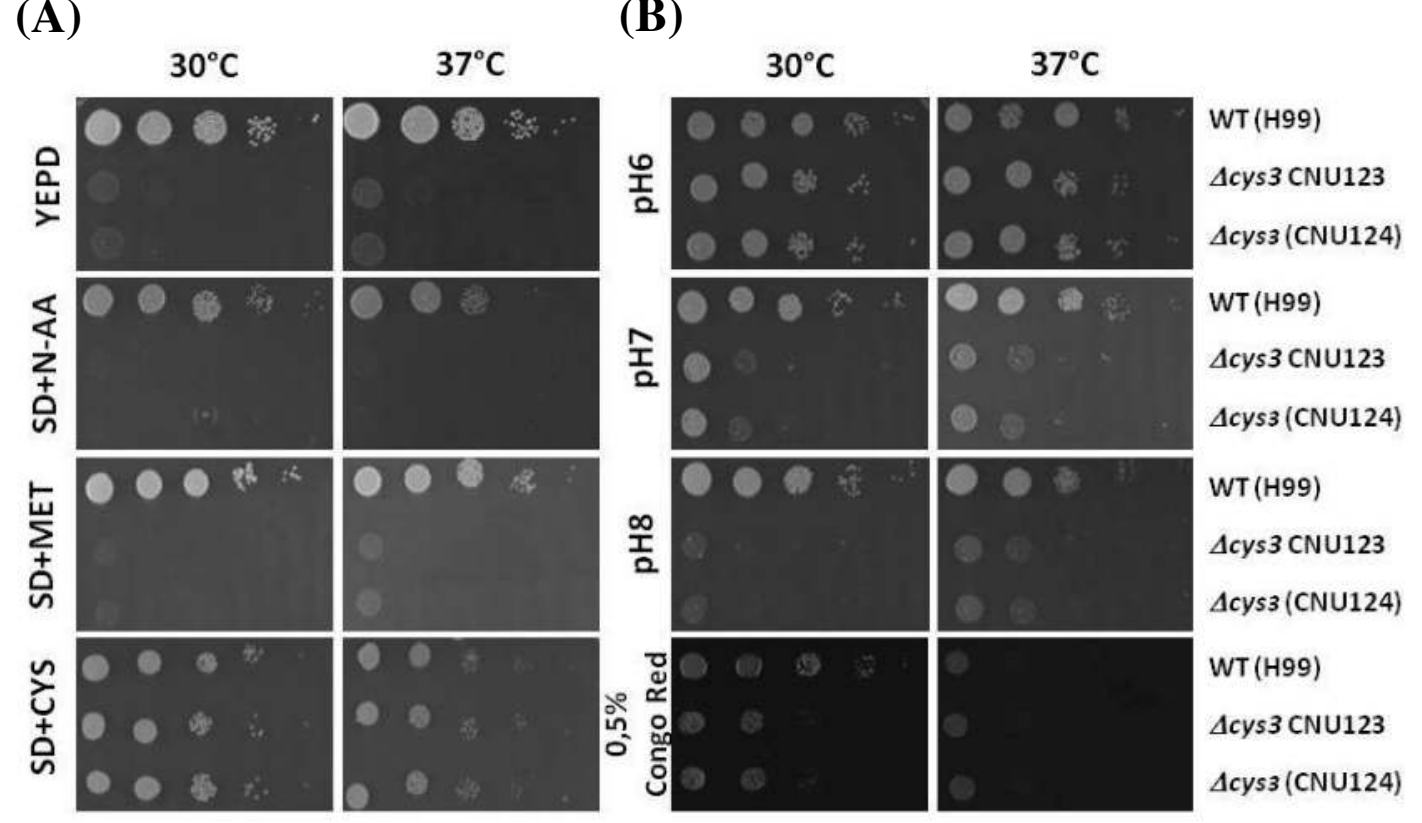

Figure 1). Growth phenotype of wild type and mutant cys $3 \Delta$ on YEPD and SD (A) and growth rate under alkaline and cell wall stress $(\mathbf{B})$.
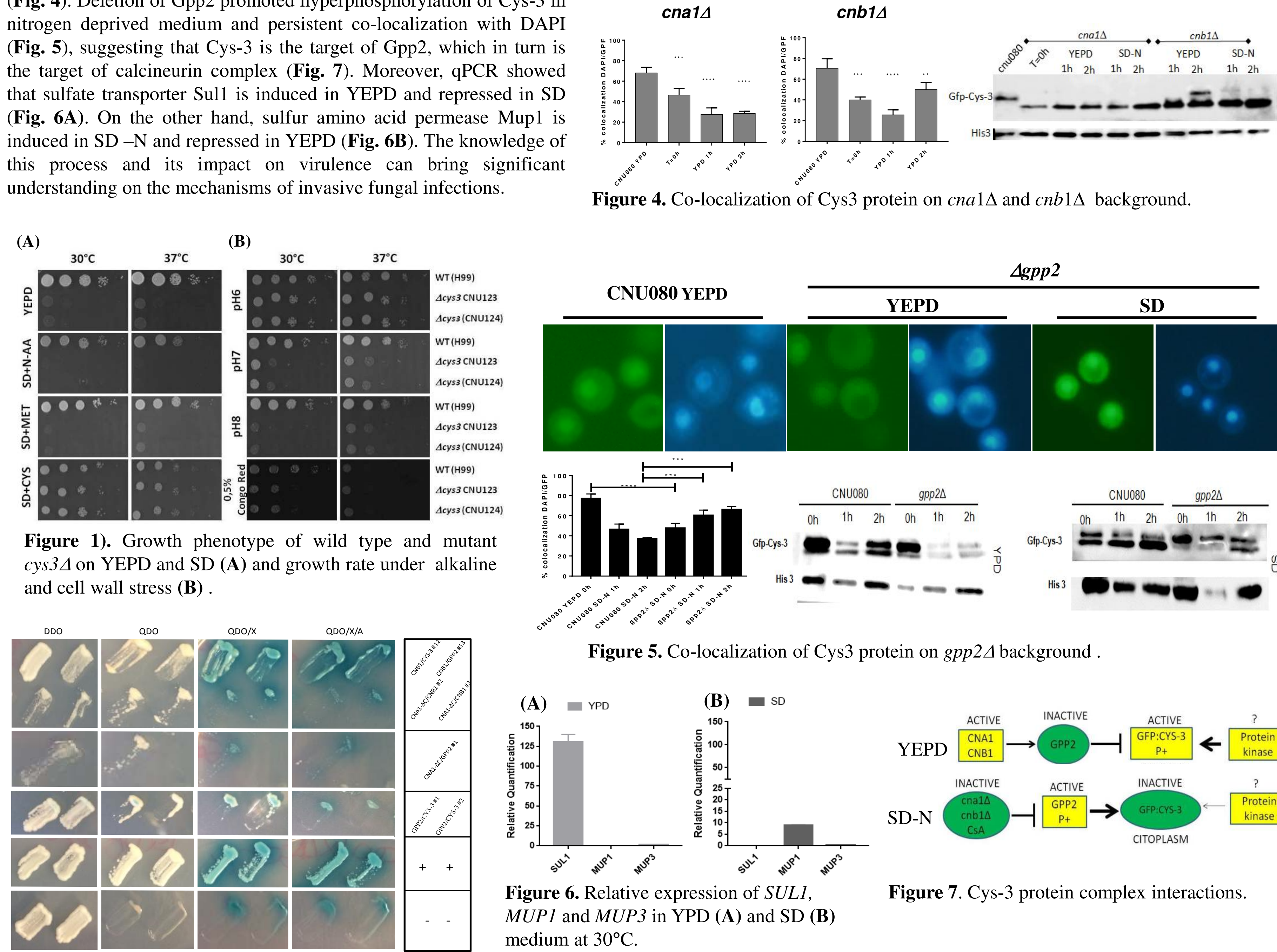

Figure 4. Co-localization of Cys 3 protein on $c n a 1 \Delta$ and $c n b 1 \Delta$ background.

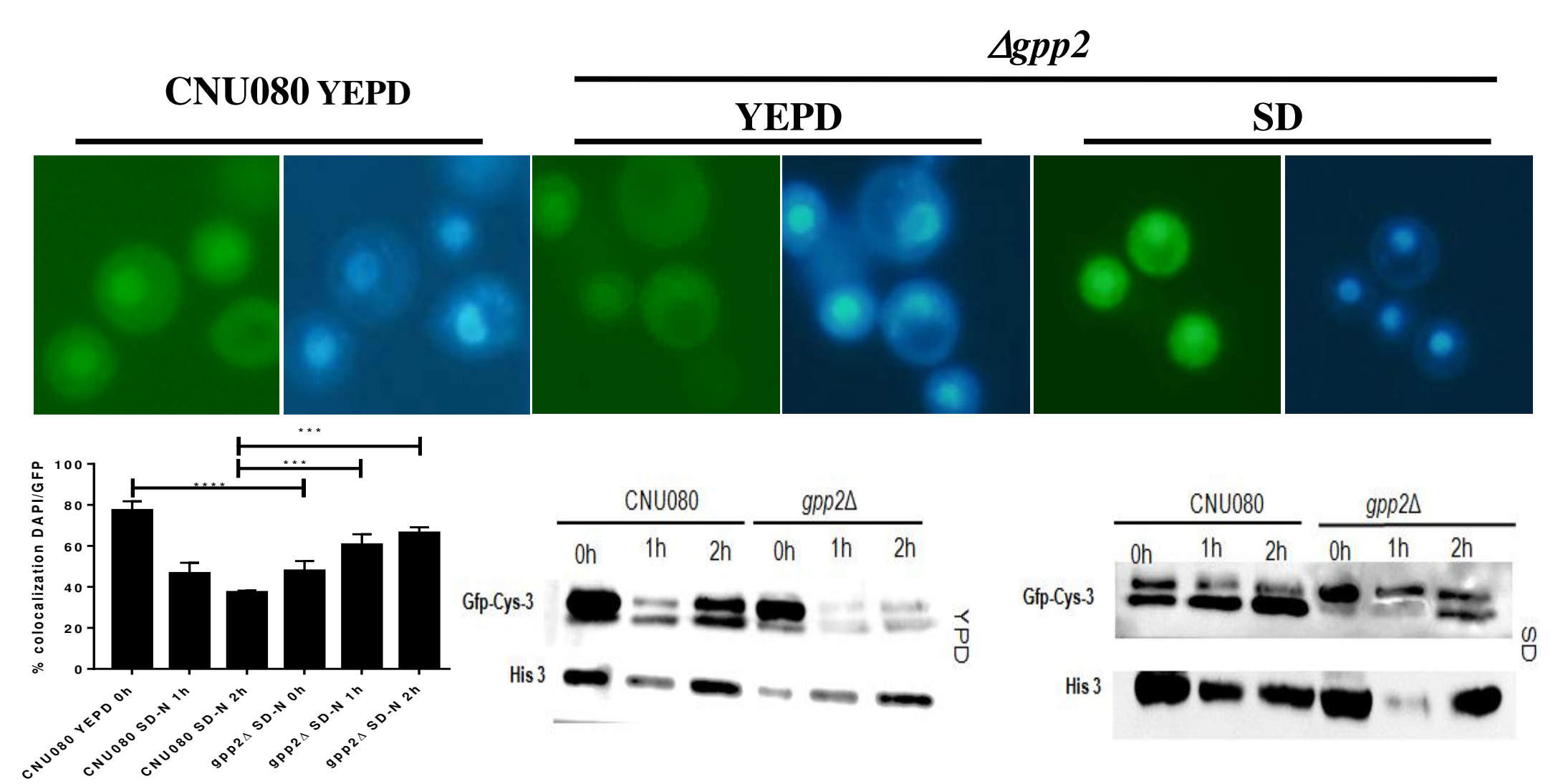

Figure 5. Co-localization of Cys 3 protein on gpp $2 \Delta$ background .
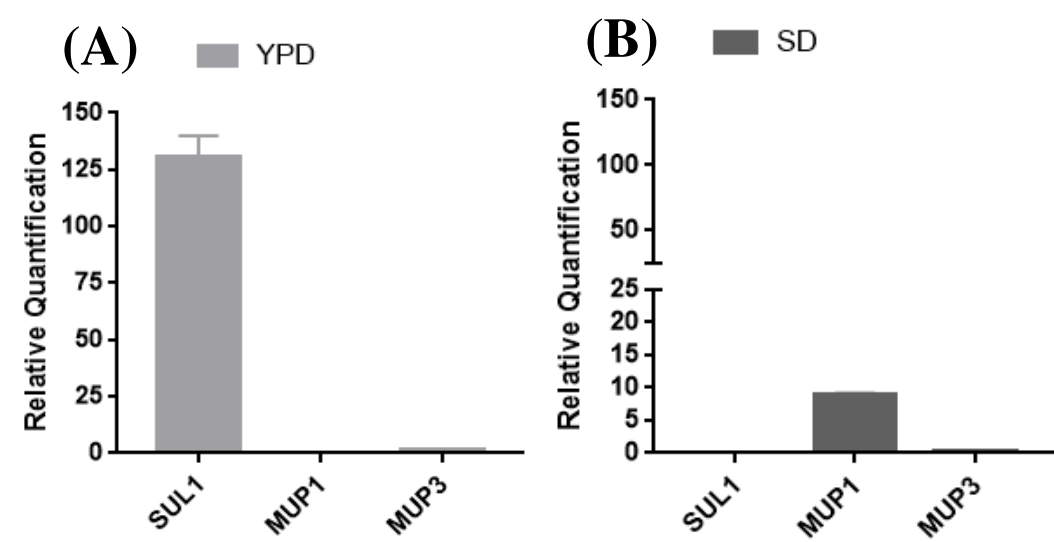

Figure 6. Relative expression of SUL1, $M U P 1$ and MUP3 in YPD (A) and SD (B) medium at $30^{\circ} \mathrm{C}$.

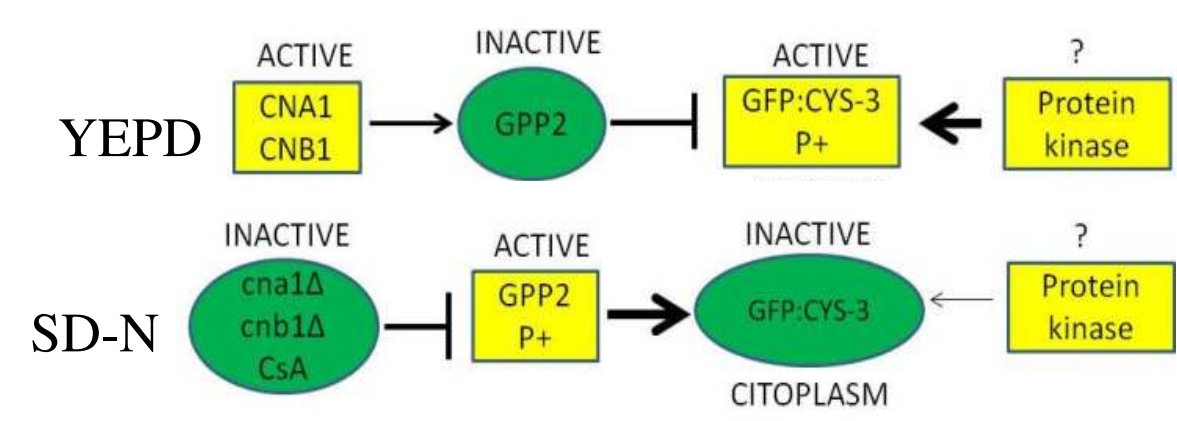

Figure 7. Cys-3 protein complex interactions. 